\title{
Design of compact multi-mode microstrip resonator filters for dual-band application
}

\author{
Nurshamimi Rosli' ${ }^{1}$, Siti Aminah Md Akhir², Siti Zuraidah Ibrahim³ , Nur Baya Mohd Hashim ${ }^{4}$, \\ Nurehansafwanah Khalid ${ }^{5}$ \\ 1,2,3,5 Advanced Communication Engineering (ACE) Centre, Universiti Malaysia Perlis, Malaysia \\ ${ }^{4}$ School of Computer and Communication Engineering, Universiti Malaysia Perlis, Malaysia
}

\begin{tabular}{l} 
Article Info \\
\hline Article history: \\
Received Aug 12, 2018 \\
Revised Nov 5, 2018 \\
Accepted Nov 26, 2018 \\
\hline
\end{tabular}

Keywords:

Bandpass filter

Dual-mode resonator

Microstrip filter

Stub-loaded resonator

\begin{abstract}
This paper presents the design of compact multi-mode microstrip resonator filters for dual-band application. The design is simulated using Advanced Design System (ADS) 2016 software. The benefit of the proposed filter is found to have a dual-mode frequency, where the odd-mode resonant frequency is the fundamental frequency represented by the length of the resonator while the even-mode resonant frequency is represented by the length of the stub. To validate the design, a prototype is fabricated on Rogers RO4003C substrate and measured using PNA Network Analyzer.
\end{abstract}

\section{Corresponding Author:}

Siti Zuraidah Ibrahim,

Advanced Communication Engineering (ACE) Centre,

School of Computer and Communication Engineering,

Universiti Malaysia Perlis,

Pauh Main Campus, 02600 Arau, Perlis, Malaysia.

Email: sitizuraidah@unimap.edu.my

\section{INTRODUCTION}

The microwave filter is a component that provides the selectivity of a frequency in mobile and satellite communication, radar and remote sensing system operating at the microwave frequency. The microstrip filter is a two port network that allows the desired frequency while attenuating the unwanted signal frequency of the filter [1-2]. Microwave multi-band especially dual-band bandpass filter becomes important component circuits in modern wireless communication system since it helps to enhance the reliability [3]. They also need to be compact and high selectivity. Multiband filter design has been seen as the other forms of multimode resonators (MMRs). MMR has an even-mode frequency and odd-mode frequency which can be analyzed in the circuits. The types of MMR include the ring resonators (RLR), stub-loaded resonator (SLR), stepped-impedance resonators (SIR) and fractal-shape resonators (FSR) [4-9]. The small size of a filter can be obtained by reducing the size of a resonator [10].

A single multi-mode resonator can extend to the multiple mode resonator filters which have a good performance and much compact size. The multimode resonator can be analyzed by using the equivalent circuit which contains two modes, which is the odd-mode and even-mode [5].

In this paper, dual-band bandpass filter using stub-loaded resonator is designed and characterized with the operating frequencies at $2.4 \mathrm{GHz}$ and $3.6 \mathrm{GHz}$. These frequencies are chosen for the application of the WiFi and WLAN by following the Institute of Electrical and Electronic Engineers (IEEE) 802.11 standard. Both frequencies are chosen as the best frequency for this design to achieve a compact size that is dependent on the 
resonator and stub length. The even-mode resonant frequency can be conveniently tuned, while the odd-mode resonant frequency remains constant.

\section{DESCRIPTION OF THE PROPOSED DESIGN}

Figure 1 shows the proposed stub-loaded resonator (SLR) which consists of a half-wavelength resonator and open stub. Y1, Y2, L1, and L2 represents the admittance and the length of the microstrip lines. Two modes, odd-mode and even-mode are used in order to analyze the proposed design filter.

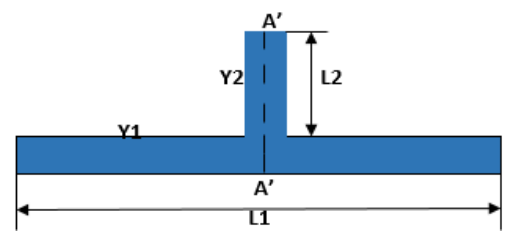

(a)

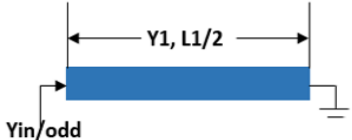

(b)

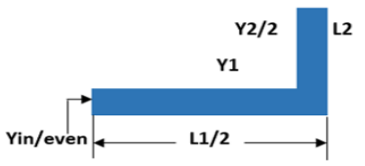

(c)

Figure 1. Structures of dual-band filter (a) The overall layout (b) Odd mode (c) Even mode

The studied design filter consists of two resonators loaded with the two stubs at the center of the respective microstrip lines. Since the stub-loaded resonator is symmetrical in structure, the odd-mode and evenmode resonant frequency can be adopted to characterize it.

\subsection{Odd-mode resonator}

There is a voltage null along the middle of the SLR. The input admittance or odd-mode, $Y_{\text {in,odd }}$ is expressed as:

$$
Y_{i n, o d d}=\frac{Y_{1}}{j \tan \left(\theta_{1} / 2\right)}
$$

where the electric length of the microstrip line is $\theta_{1}=\beta L_{1}$. Since $Y_{\text {in }, \text { odd }}=0$,

$$
f_{\text {odd }}=\frac{(2 n-1) c}{2 L_{1} \sqrt{\varepsilon_{\text {eff }}}}
$$

where $n=1,2,3 \ldots$ In this paper, $n=1$ is chosen since a higher value of $n$ will lead to the large size of the filter. Thus, $n=1$ is used to maintain the compactness of the design filter.

\subsection{Even-mode resonator}

At the even-mode, there is no current flow through the symmetrical plane. The result of the input admittance for even-mode $Y_{\text {in even }}$ can be expressed as:

$$
Y_{\text {in,even }}=j Y_{1} \frac{2 Y_{1} \tan \left(\theta_{1} / 2\right)+Y_{2} \tan \left(\theta_{2}\right)}{2 Y_{1}-Y_{2} \tan \left(\theta_{1} / 2\right) \tan \left(\theta_{2}\right)}
$$

where $\theta_{2}=\beta L_{2}$ is the open stub electric length. Since $Y_{\text {in,even }}=0$, the even-mode resonant frequency is:

$$
\cot \left(\theta_{1} / 2\right) \tan \left(\theta_{2}\right)=-\frac{2 Y_{1}}{Y_{2}}
$$

For the special case of $Y_{2}=2 Y_{1}$

$$
\cot \left(\theta_{1} / 2\right) \tan \left(\theta_{2}\right)=-1
$$

Therefore, the resonance condition for this case is: 
or

$$
\theta_{1} / 2+\theta_{2}=n \pi
$$

$$
L_{1} / 2+L=n \lambda_{g} / 2
$$

where $\lambda_{g}$ is the guided wavelength at even-mode resonance. The frequency at the even-mode, $L_{2}$ can be obtained from equation (8):

$$
f_{\text {even }}=\frac{n c}{\left(L_{1}+2 L_{2}\right) \sqrt{\varepsilon_{e f f}}}
$$

To verify the above analysis, full-wave EM simulation has been carried out. The dual-mode resonator is designed to achieve the desired resonant frequencies. The required frequency is fixed by the length of resonator, $L_{1}$ and the even-mode resonant frequency can be achieved by adjusting the length of the stub, $L_{2}$. Two microstrip line with $50 \Omega$ characteristic impedance is utilized to feed the proposed SLR filter using a loose coupling. The length of the $L_{l}$ is fixed at $35.3 \mathrm{~mm}$, while $L_{2}$ is varied from $1 \mathrm{~mm}$ to $7 \mathrm{~mm}$ as shown in Figure 2. The value of $5.8 \mathrm{~mm}$ is chosen as the best stub length for this design and labeled with a blue color.

As can be seen in Figure 2, the stub-length does not affect the $S_{21}$ response at an odd-mode frequency at $2.4 \mathrm{GHz}$, whereas the even-mode frequency can be shifted by controlling the value of $L_{2}$. The higher the value of $L_{2}$, the lower the value of $f_{\text {even }}$.

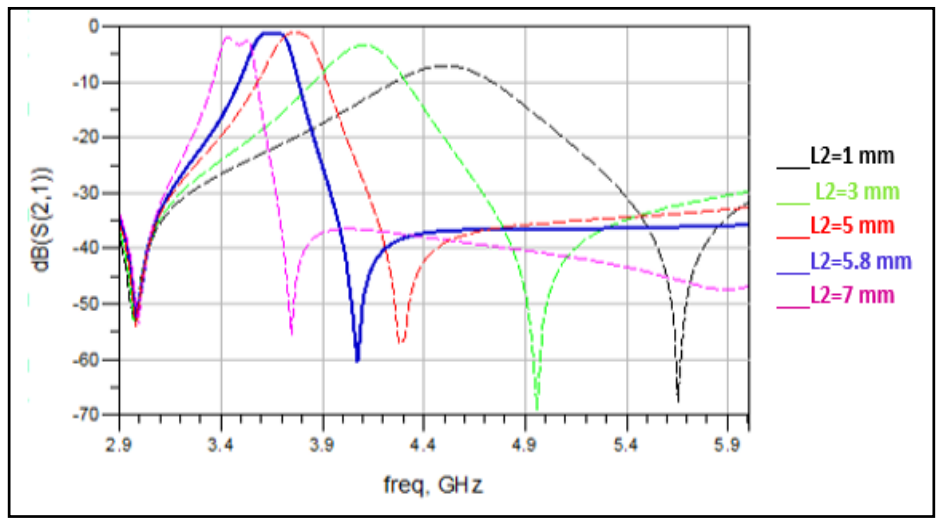

Figure 2. Simulated parameter with different stub length

\section{DUAL-BAND FILTER DESIGN}

Based on proposed SLR, a dual-band bandpass filter which has a benefit of compact size is designed. The configuration of the filter which consists of two folded SLRs is shown in Figure 3 while Figure 4 shows the fabricated dual-band filter. The Rogers RO4003C is used as the substrate for this design which has a relative dielectric constant, $\varepsilon_{r}$ of 3.38, a thickness of $0.508 \mathrm{~mm}$ and the dissipation factor, $\tan \delta$ of 0.0027 . The dimensions of the proposed filter are shown in Table 1.

Table 1. Dimensions of the Filter

\begin{tabular}{cc}
\hline Parameters & Value $(\mathrm{mm})$ \\
\hline Length of resonator, $L_{1}$ & 35.3 \\
Length of stub, $L_{2}$ & 5.8 \\
Width of resonator, $W_{1}$ & 0.9 \\
Width of stub, $W_{2}$ & 1.8 \\
Distance, $d$ & 0.6 \\
Gap, $S_{1}$ and $S_{2}$ & 0.3 \\
\hline
\end{tabular}

According to expression (1) to (8), the length of the resonator and stub can be obtained where $f$ is the center frequency of the filter and $c$ is the speed of light. The effective dielectric constant, $\varepsilon_{\text {eff }}$ and ratio W/d is calculated from the following expression. 


$$
\begin{aligned}
& \varepsilon_{\text {eff }}=\frac{\varepsilon_{r+1}}{2}+\frac{\varepsilon_{r-1}}{2}\left(1+12 \frac{d}{W}\right)^{-\frac{1}{2}} \\
& \frac{W}{d}=\frac{8 e^{A}}{e^{2 A}}
\end{aligned}
$$

The simulation is done by using the EM simulation in the ADS software and measured with the PNA network Analyzer, respectively.

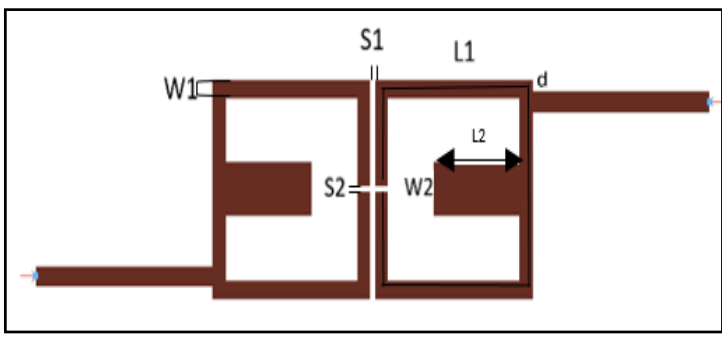

Figure 3. Layout of dual-band filter

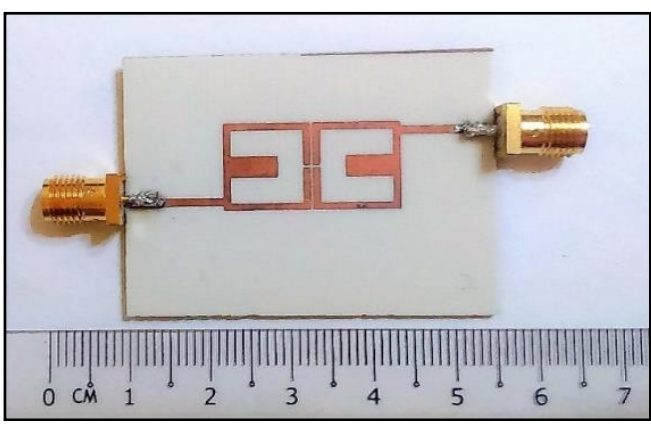

Figure 4. Fabricated dual-band filter

\section{RESULTS}

Figure 5 and 6 illustrates the simulated and measured result of the S-parameters respectively to the studied dual-band bandpass filter which operated at two central frequencies, $2.4 \mathrm{GHz}$, and 3.6 GHz. Based on the simulated result, it shows that the both frequency is located at the $2.4 \mathrm{GHz}$ and $3.6 \mathrm{GHz}$ respectively with a small frequency shifting is observed.

From the calculation, it can be analyzed that the guided wavelength, $\lambda_{g}$ as highlighted below is depending on the value of dielectric constant of the substrate. Meanwhile, the value of the relative permittivity, $\varepsilon_{r}$ will affect the effective dielectric constant.

$$
\lambda_{g}=\frac{c}{f \sqrt{\varepsilon_{e f f}}}
$$

Consistent with the design filter, the observed fractional bandwidth of measured result is $8 \%$ and $6 \%$ while the simulated fractional bandwidth is $7 \%$ and $5 \%$. The reflection coefficient, $\mathrm{S}_{11}$ is decreased compared to the simulated result which is $-11.94 \mathrm{~dB}$ and $-14.51 \mathrm{~dB}$. There are two transmission zeroes at the second frequency of simulated result compared to no transmission zeroes at the measured result. The reflection coefficient for the second frequency of simulated result is decreased from $-25.47 \mathrm{~dB}$ to $-26.99 \mathrm{~dB}$. The reflection coefficient performance of both results is satisfied.

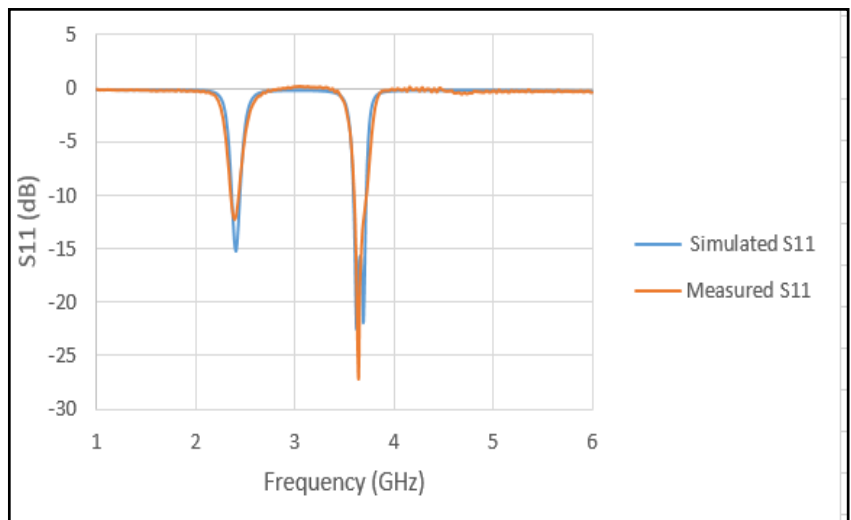

Figure 5. Simulated and measured $S_{11}$ parameters of dual-band filter 
Next, the transmission coefficients, $S_{21}$ of simulated and measured results are illustrated in Figure 6. The simulated transmission coefficient at the first frequency is $-1 \mathrm{~dB}$ and $-1.24 \mathrm{~dB}$ while the measured result has transmission coefficient at $-1.45 \mathrm{~dB}$ and $-1.96 \mathrm{~dB}$. The transmission coefficient in the measured result is decreased with $0.45 \mathrm{~dB}$. The measured transmission coefficient is degraded compared to the simulated results because of the fabrication tolerances.

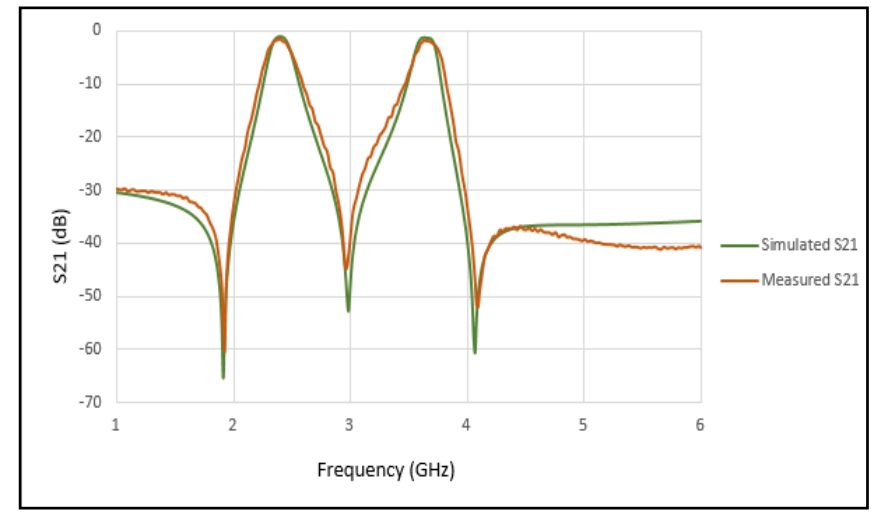

Figure 6. Simulated and measured $S_{21}$ parameters of dual-band filter

The bandwidth for the simulated result at $2.4 \mathrm{GHz}$ and $3.6 \mathrm{GHz}$ is $170 \mathrm{MHz}$ and $187 \mathrm{MHz}$ and the bandwidth for the measured result at is $200 \mathrm{MHz}$ and $225 \mathrm{MHz}$. The simulated fractional bandwidth is 0.07 at the first frequency and 0.05 at the second frequency. The measured fractional bandwidth for odd-mode frequency is 0.08 and 0.06 at the even-mode frequency which is second frequency. Note that the even-mode, $f_{\text {even }}$ is controlled by varying the value of the stub length, $L_{2}$ while the odd-mode, $f_{\text {odd }}$ can be dominated by varying the resonator length, $L_{l}$ which can be calculated from expression (2).

\section{CONCLUSION}

In this paper, a multi-mode technique to obtain dual-band filter, SLR is proposed. The properties of the SLR are obtained by simulation analysis in the ADS software and verified experimentally by fabricating and measuring the $\mathrm{S}$-parameters of the prototype. Besides having an advantage on its compact size, the resonant frequency of even-mode can be controlled by varying the length of stub while the odd-mode resonant frequency is kept constant. The measured results were agreed well with the simulated result.

\section{ACKNOWLEDGEMENTS}

The author would like to acknowledge the support from the Fundamental Research Grant Scheme (FRGS) under a grant number of FRGS/1/2015/TK04/UNIMAP/02/16 from the Ministry of Higher Education Malaysia.

\section{REFERENCES}

[1] David M. Pozar, (2011), Microwave Engineering, 4th Edition, John Wiley \& Sons, Inc USA.

[2] R. A. Rahim, et al., "Microstrip dual-band bandpass filter for ISM band applications," 2015 IEEE International Conference on Control System, Computing and Engineering (ICCSCE), George Town, 2015, pp. 281-286.

[3] H. -Y Anita Yim. et al., "A New Synthesis Method for Dual-Band Microwave Filter Design with Controllable Bandwidth," 2007 Asia-Pacific Microwave Conference, Bangkok, 2007, pp. 1-4.

[4] M. Pal and R. Ghatak, "A Distinctive Resonance: Multiband Bandpass Filter Design Techniques Using Multimode Resonators," IEEE Microwave Magazine, vol. 16, no. 11, pp. 36-55, Dec. 2015.

[5] X. Y. Zhang, et al., "Dual-Band Bandpass Filters Using Stub-Loaded Resonators," IEEE Microwave and Wireless Components Letters, vol. 17, no. 8, pp. 583-585, Aug. 2007.

[6] P. Sarkar, et al., "A Planar Compact Dual-Band Bandpass Filter Using Stepped Impedance Resonator and Interdigital Capacitor, " Int. J. Microw. Wirel. Technol., vol. 3, no. 6, pp. 671-674, 2011.

[7] J. Z. Chen, et al., "Fourth-order Tri-band Bandpass Filter Using Square Ring Loaded Resonators, " Electron. Lett., vol. 47, no. 15, pp. 858-U1942, 2011. 
[8] S. Neethu and S. S. Kumar, "Microstrip Bandpass Filter Using Fractal-Based Hexagonal Loop Resonator," Proc. 2014 4th Int. Conf. Adv. Comput. Commun. ICACC 2014, pp. 319-322, 2014.

[9] M. Mabrouk and L. Bousbia, "Study and Enhanced Design of RF Dual Band Bandpass Filter Validation and Confirmation of Experimental Measurements," Circuit and Systems, vol. 2, pp. 293-296, 2011.

[10] K. Ma, et al., "A Compact Multimode Bandpass Filter With Extended Stopband Bandwidth," Progress in Electromagnetics Research Letters, vol. 32, pp. 177-186, 2012.

\section{BIOGRAPHIES OF AUTHORS}

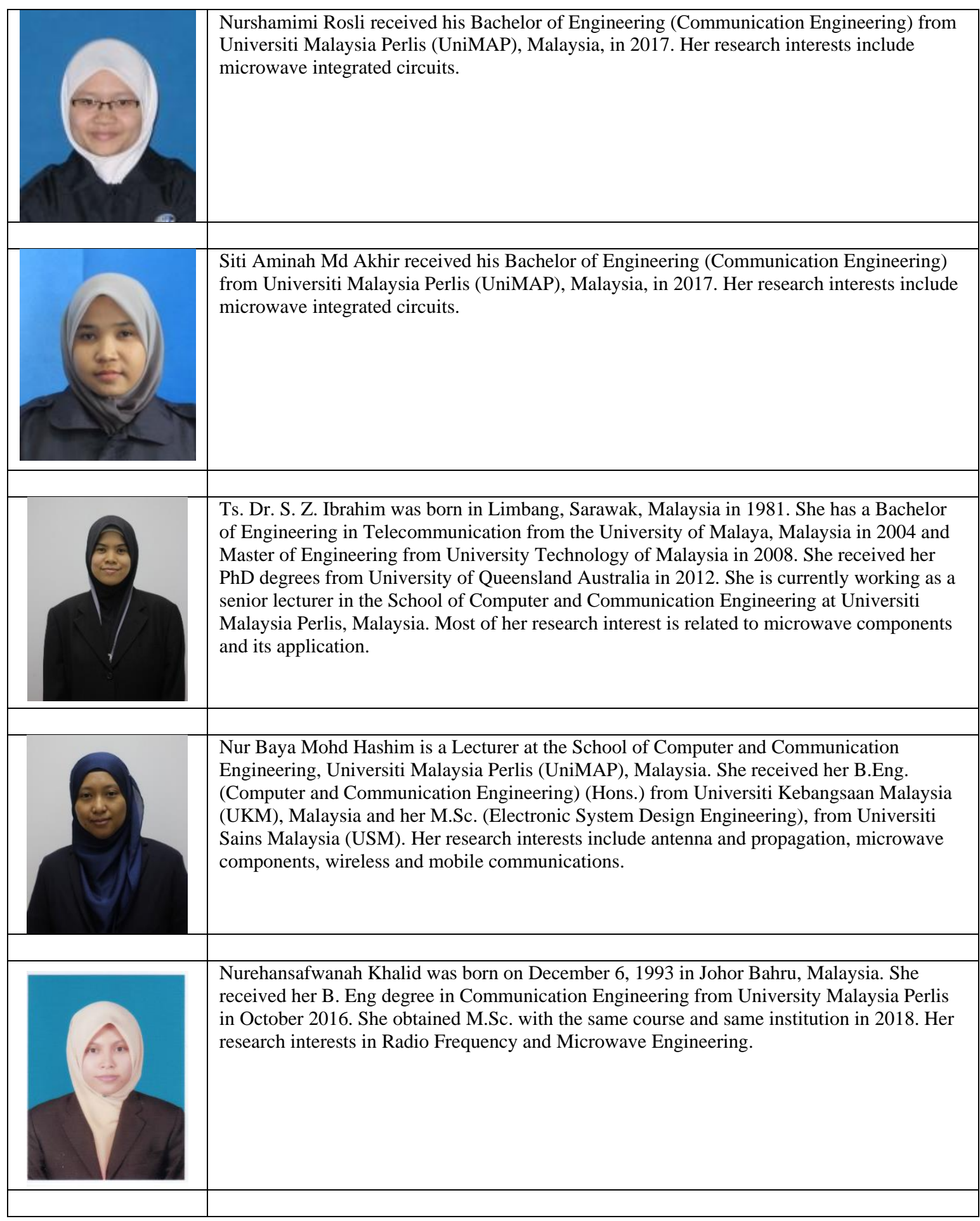

